\title{
Beam characterization for the field-emitter-array cathode-based low-emittance gun
}

\author{
S. C. Leemann, ${ }^{*}$ A. Streun, and A.F. Wrulich \\ Paul Scherrer Institute, CH-5232 Villigen PSI, Switzerland
}

(Received 16 March 2007; published 12 July 2007)

\begin{abstract}
A $1 \AA$ x-ray free electron laser can be operated at $6 \mathrm{GeV}$ provided a very high brightness electron beam. Therefore, Paul Scherrer Institute has initiated development of the low-emittance gun based on field emission. A field-emitter array (FEA) is expected to deliver high peak current while reducing the source emittance to levels close to the thermal limit. A $100 \mathrm{keV}$ gun test stand has been designed in order to gain experience with electron bunches emitted by pulsed FEAs, investigate emittance compensation, and develop diagnostic procedures to characterize the beam. First FEA samples have been installed and pulsed in the gun; the emitted bunches have been accelerated and characterized. We present results acquired with the first FEA samples.
\end{abstract}

DOI: 10.1103/PhysRevSTAB.10.071302

PACS numbers: 29.27.Fh, 85.45.Db, 29.25.Bx, 41.75.Ht

\section{INTRODUCTION}

In the quest for higher brightness synchrotron radiation, the first 4 th generation sources are presently under construction. These sources are based on $\mathrm{X}$-ray free electron lasers (X-FEL) where intense high energy electron beams generate short-pulsed, spatially and temporally coherent radiation with wavelengths as low as $1 \AA$. The most advanced projects are the LCLS at SLAC [1] and the European XFEL at DESY [2]. These facilities require long linac sections to accelerate the short and intense electron bunches to 14 and $20 \mathrm{GeV}$, respectively. Paul Scherrer Institute (PSI) is interested in a $1 \AA \mathrm{X}$-FEL as a companion light source to the existing 3rd generation Swiss Light Source [3]. However, compared to other $1 \AA$ $\mathrm{X}$-FEL projects, the PSI facility needs to be scaled down considerably to fit site and budget constraints. It is therefore crucial to develop a concept which reduces the required beam energy given by the desired radiation wavelength $\lambda$ according to the spatial coherence criterion

$$
\frac{\varepsilon^{(n)}}{\beta \gamma}<\frac{\lambda}{4 \pi}
$$

where $\varepsilon^{(n)}$ is the normalized transverse emittance at the undulator entrance. Provided a normalized transverse emittance of $10^{-7} \mathrm{~m} \mathrm{rad}, 1 \AA$ radiation can be generated by an $\mathrm{X}$-FEL running at $6 \mathrm{GeV}$ [4-6].

The challenge is therefore to design an accelerator which provides electron bunches with a normalized transverse emittance which is roughly 10 times lower than what is required by other $1 \AA \mathrm{X}$-FEL projects. Since the normalized transverse emittance in a perfect linac is a conserved quantity, this translates to finding an electron source with unprecedentedly low emittance. Furthermore, fast

*simon.leemann@psi.ch; http://slsbd.psi.ch/ leemann acceleration in a high-gradient gap should reduce emittance blowup due to space-charge forces at low energy. Assuming homogeneous emission from a flat cathode, there is a fundamental emittance minimum given by the thermal energy of the emitted electrons. A simple calculation shows that the normalized transverse source emittance can be expressed by

$$
\varepsilon^{(n)}=\frac{\gamma r_{c}}{2} \sqrt{\frac{k_{B} T}{m_{e} c^{2}}},
$$

where $r_{c}$ is the cathode radius and $T$ is the temperature of the cathode. For thermionic cathodes, reducing the source emittance to the desired levels requires a reduction of the cathode size. However, since the undulator requires a peak current of $1.5 \mathrm{kA}$ which (assuming strong magnetic bunch compression) translates to several amperes of peak current from the gun [4-6], a small thermionic cathode is ruled out. A photocathode offers the advantage of emitting short electron bunches and operating at room temperature. For operation in a high-gradient gap, the cathode should not have a low work function to prevent dark current. However, if a cathode with a large work function is used, a short wavelength laser is required to draw sufficient current. This leads to a large transverse momentum spread of the emitted electrons which translates to an increase of source emittance.

\section{LOW-EMITTANCE GUN}

Because of the previously mentioned shortcomings of present-day electron guns, PSI has started development of the low-emittance gun: a novel type of electron gun capable of supplying $5.5 \mathrm{~A}$ of peak current with a transverse emittance of $5 \times 10^{-8} \mathrm{~m} \mathrm{rad}$ in $40 \mathrm{ps}$ bunches [5,6]. For operation at room temperature, the cathode size is therefore limited to $r_{c}<0.45 \mathrm{~mm}$. As proposed by Tang et al. [7], a field-emitter array (FEA) is an ideal candidate since 
it delivers both high current density and low source emittance. In such a FEA thousands of $\mu \mathrm{m}$-sized metallic cones are distributed regularly over the cathode surface. A $1 \mu \mathrm{m}$ thin dielectric layer separates the cones from the gate layer which acts as a control electrode. If a moderate voltage pulse is applied to the gate layer, field enhancement at the tip of the cone leads to peak electric field strengths of several $\mathrm{GV} / \mathrm{m}$ which trigger field emission of electrons into vacuum. Double-gated FEAs have an additional control electrode above the gate layer, the so-called focusing layer. By applying an appropriate voltage between the gate layer and the focusing layer, the beamlet emerging from each tip is electrostatically focused. By minimizing the divergence of each beamlet, the overall source emittance is minimized. In the PSI low-emittance gun a double-gated FEA cathode will be installed in a $4 \mathrm{~mm}$ high-voltage (HV) gap. A $1 \mathrm{MV}$ pulse is applied to the cathode for $200 \mathrm{~ns}$. During this pulse a secondary moderate voltage pulse is applied to the FEA gate layer which triggers field emission from the FEA tips. The emitted electrons are accelerated to $1 \mathrm{MeV}$ in the HV gap. The bunch then enters the twofrequency S-band rf cavity where it is further accelerated to $4 \mathrm{MeV}$. Several solenoids are installed for focusing and emittance compensation. Ballistic compression of the bunch is finalized by a $1.5 \mathrm{GHz}$ traveling wave structure. At $30 \mathrm{MeV}$ the bunch enters the main linac section with its two magnetic bunching chicanes. The bunch is accelerated to $6 \mathrm{GeV}$ and compressed to a length of $50 \mathrm{fs}$ giving a peak current of $1.5 \mathrm{kA}$; the normalized transverse slice emittance is expected to be $0.1 \mathrm{~mm} \mathrm{mrad}$. The bunch then passes eight $4.5 \mathrm{~m}$ long undulators with $15 \mathrm{~mm}$ period length and $6 \mathrm{~mm}$ gap where the coherent $1 \AA$ radiation is generated [4].

\section{EXPERIMENTAL SETUP}

A $100 \mathrm{keV}$ gun test stand has been designed and commissioned in order to gain first experience with highgradient acceleration of electron bunches from FEAs, investigate emittance compensation with a solenoid magnet, and develop diagnostic procedures to characterize lowemittance bunches. A modular design was chosen in order to facilitate exchange of components. The FEA is installed behind the removable cathode electrode. The entire cathode assembly and FEA pulser are put on a DC potential of $-100 \mathrm{kV}$. The pulser delivers 5-100 ns long pulses with amplitudes from 0-320 V. The grounded anode electrode and in-vacuum solenoid magnet are inserted in the other end of the ceramic break. The gun and solenoid were designed for $100 \mathrm{~mA}$ peak current bunches emitted from a $1 \mathrm{~mm}$ diameter FEA. The electrostatic and magnetostatic modeling as well as particle tracking was done with the code MAFIA in 2.5D [8]. By choosing a small in-vacuum solenoid magnet with a high-permeability yoke, a bucking coil behind the cathode was not required. Alignment tolerance studies were performed in 3D with the code GPT [9].

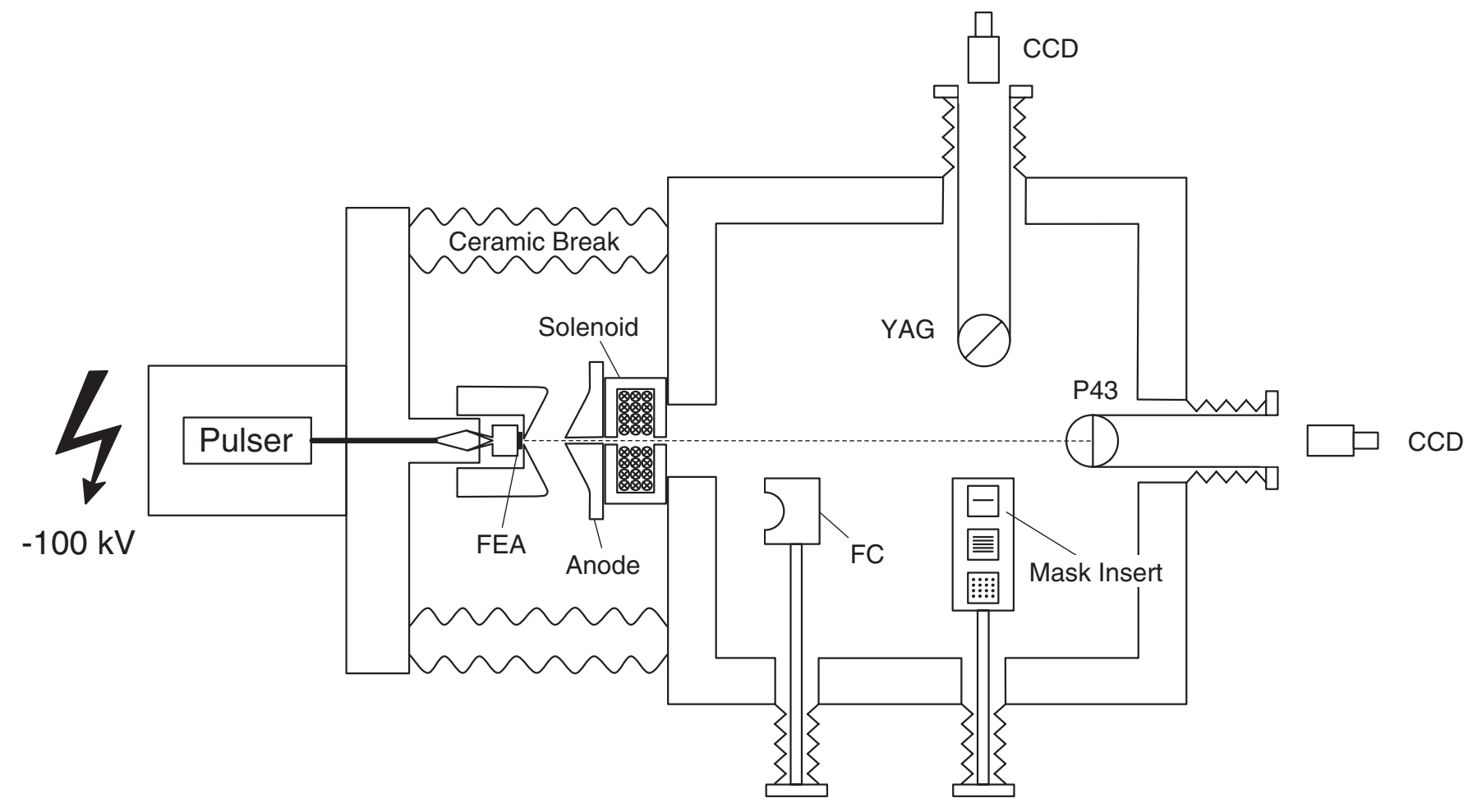

FIG. 1. Schematic overview of the $100 \mathrm{keV}$ gun test stand (not to scale). 
Downstream of the solenoid magnet the diagnostics module is attached. It holds a Faraday cup, horizontal and vertical mask inserts, and two screen monitor systems (YAG and P43 phosphor). The point-spread function of the screen monitor optics has been determined to be approximately $3 \mu \mathrm{m}$ which is on the order of the granularity of the phosphor coating and hence sufficient. Each mask insert carries a single slit, slit array, and pinhole mask. Particle tracking through the masks and up to the screen monitors was performed with MAFIA in order to specify mask geometries optimized to resolve emittances lower than a few $10^{-6} \mathrm{~m}$ rad with an accuracy of several percent. A schematic overview of the $100 \mathrm{keV}$ gun test stand is given in Fig. 1.

\section{MEASUREMENTS AND RESULTS}

For commissioning of the test stand commercially available FEAs from SRI International [10] were used. These FEAs are gated but do not have a focusing layer. Roughly 50000 conical molybdenum tips form a $1 \mathrm{~mm}$ diameter active emitting area on the silicon substrate. Several FEAs of this type have been installed at the test stand.

\section{A. FEA performance and lifetime}

The emission current shows a very strong dependence on gate voltage as predicted by the Fowler-Nordheim law [11]. However, the HV determines the level at which emission current saturates due to screening of the cathode (Child's Law) [12]. The FEAs are extremely sensitive to HV breakdown. Ion back bombardment can destroy single tips or entire clusters of tips depending on the accelerating $\mathrm{HV}$. At an accelerating $\mathrm{HV}$ of $40 \mathrm{kV}$ most of the FEA samples could be operated stably up to a gate voltage of roughly $200 \mathrm{~V}$. An RC constant of roughly 10 ns was

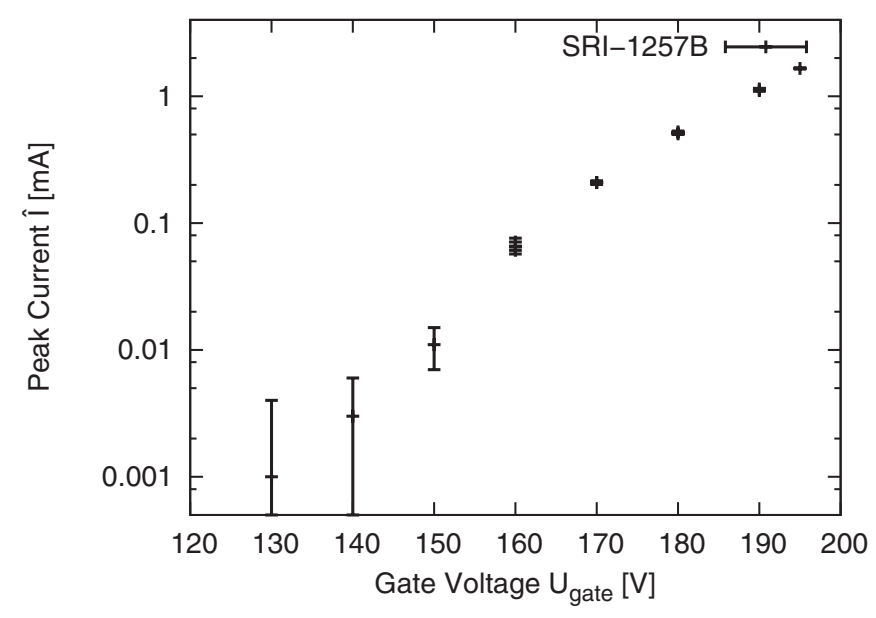

FIG. 2. FEA performance calibration using $40 \mathrm{kV}$ accelerating $\mathrm{HV}$, a pulse length of $100 \mathrm{~ns}$, and a repetition rate of $100 \mathrm{~Hz}$. Above $200 \mathrm{~V}$ emission became unstable and triggered HV breakdown. determined for the FEA assembly. Applying the maximum pulse length of $100 \mathrm{~ns}$, this allowed emission of bunches with up to $100 \mathrm{pC}$ and with a maximum peak current of roughly $2 \mathrm{~mA}$ (see Fig. 2). Images of unfocused bunches showed hot spots and nonuniformities due to damage of the emitting surface leading to inhomogeneous emission.

\section{B. Transverse beam characterization}

Since the SRI FEAs were suspected of emitting emittance dominated beams rather than space-charge dominated beams, the emittance was first measured with a solenoid scan [6]. The rms beam size data was fitted to the applied solenoid current which rendered the emittance and Courant-Snyder parameters. An example for a solenoid scan is given in Fig. 3. The solenoid scan results were compared to the emittance measured with the single slit method: The rms beam size was first measured with the YAG screen at the exact location of the single slit mask. The width of the beamlet emerging from the single slit was then measured with the phosphor screen monitor for several different drift distances. This gives the uncorrelated beam divergence spread at the location of the slit. An example for such a measurement is given in Fig. 4. Together with the previously measured rms beam size the emittance can be derived. The resulting horizontal emittance of $(2.41 \pm 0.19) \mathrm{mm}$ mrad is perfectly compatible with the result given by the solenoid scan: (2.61 \pm $0.18) \mathrm{mm}$ rad. This emittance result together with the measured peak current and beam size confirm that the SRI FEAs produce emittance dominated bunches.

The emittance measurements were further compared to measurement results acquired with the pinhole array. The pinhole array mask was inserted into the beam and the emerging beamlets imaged on the $\mathrm{P} 43$ phosphor screen. A screen monitor image of the pinhole array beamlets is shown in Fig. 5. An example of the phase space distribution density reconstruction [6] is given in Fig. 6. The emittance

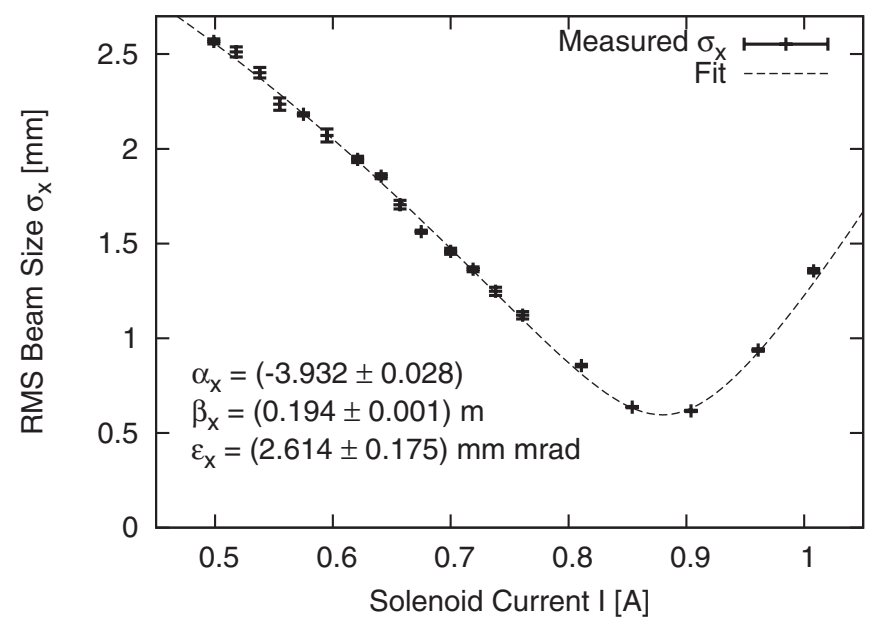

FIG. 3. Solenoid scan data and fit in the horizontal plane. The derived results have been calculated at the solenoid entry yoke. 

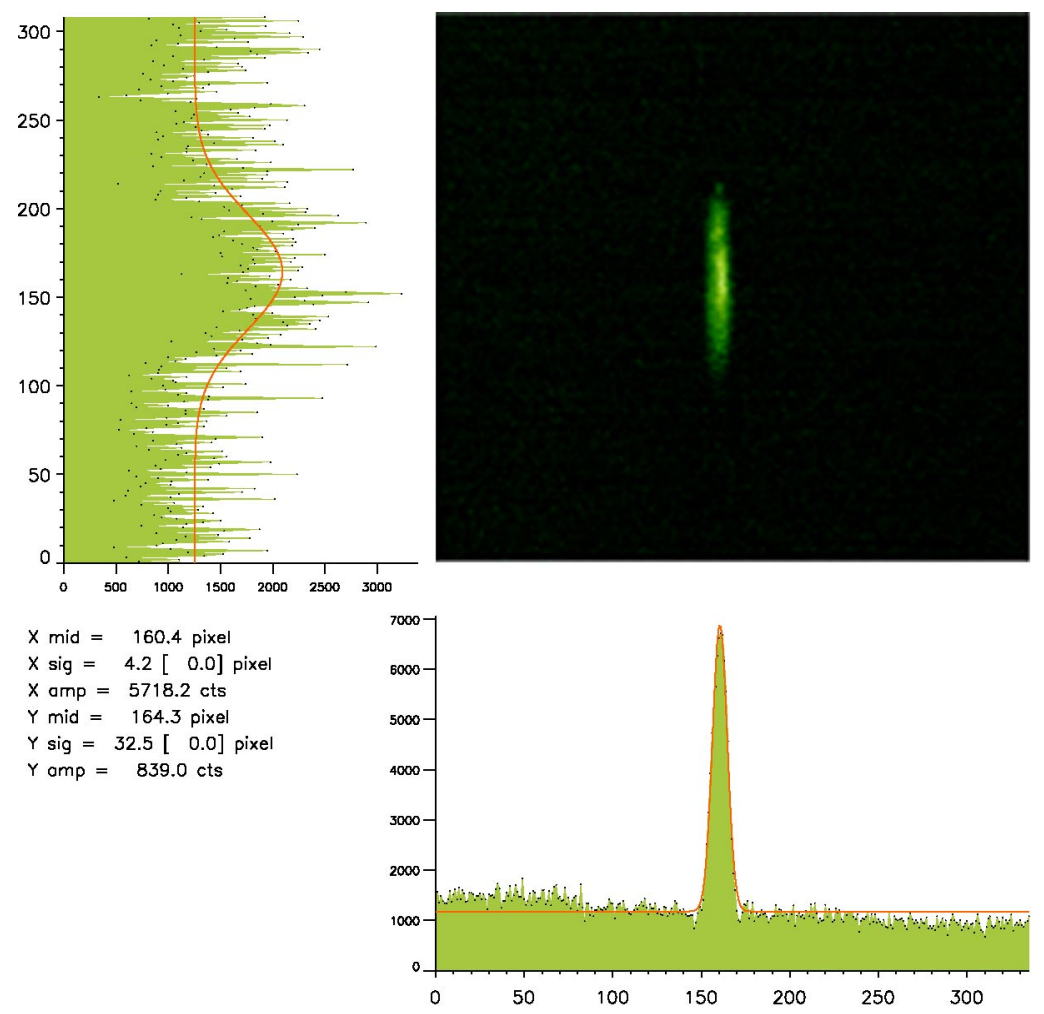

FIG. 4. (Color) Single slit emittance measurement. The beamlet was imaged on a P43 phosphor screen. The histograms show intensity in arbitrary units vs pixel number. The beamlet rms width measured here is $\sigma_{x}=(0.138 \pm 0.007) \mathrm{mm}$. This corresponds to an uncorrelated beam divergence spread of $\sigma_{x^{\prime}}=(3.54 \pm 0.20) \mathrm{mrad}$ at the slit location.
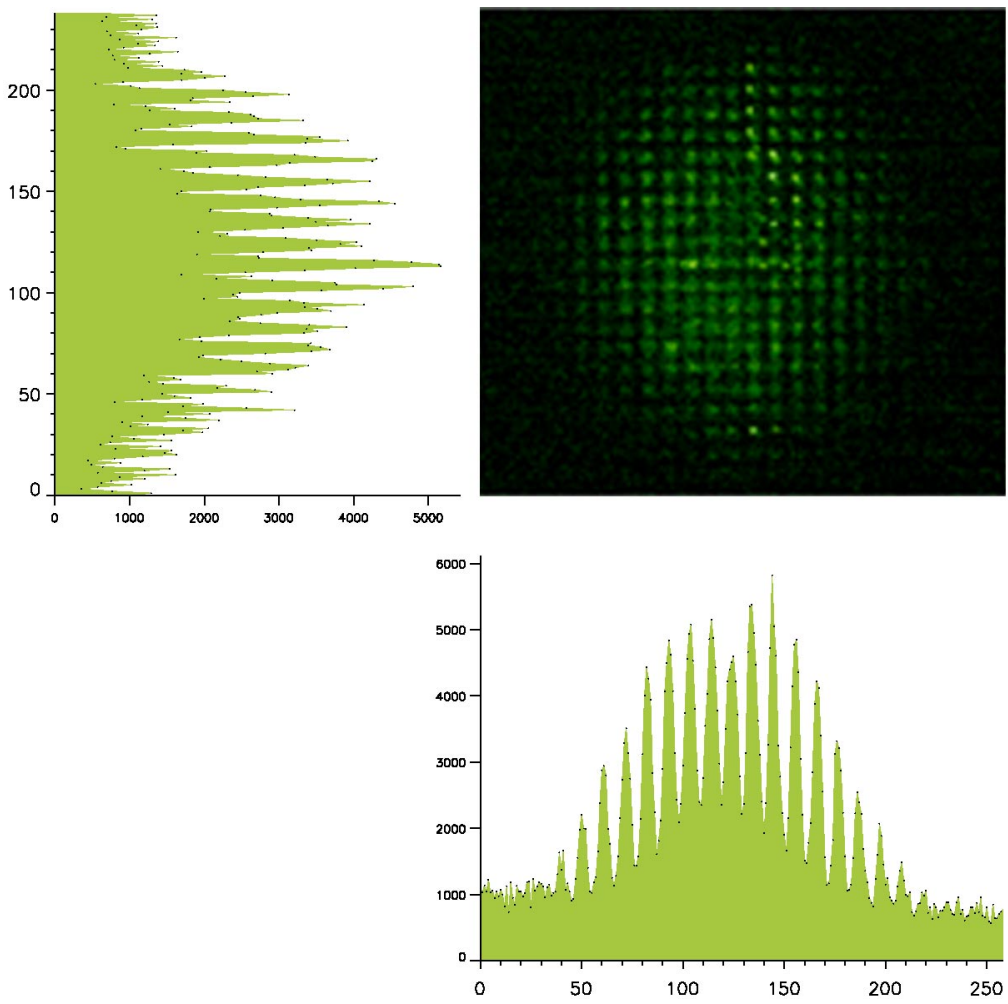

FIG. 5. (Color) Pinhole array measurement. The beamlets were imaged on a P43 phosphor screen. The histograms show intensity in arbitrary units vs pixel number. 


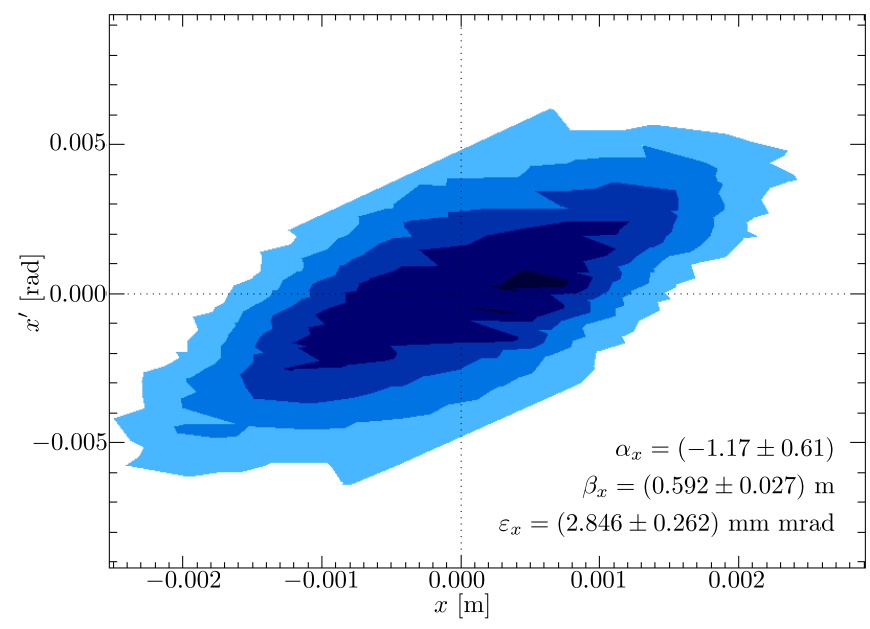

FIG. 6. (Color) Horizontal phase space density reconstruction from pinhole array data. The image and printed results were calculated using the data shown in Fig. 5.

and Courant-Snyder parameter results are in agreement for all applied measurement methods.

The emittance does not increase with bunch charge as expected for an emittance dominated beam; the emittance did however increase after HV breakdown incidents (see Fig. 7). Contamination of the FEA and damage to the gate and tip layer lead to nonuniform emission which can increase the source emittance considerably [13,14]. A transition from the emittance to the space-charge dominated beam can be expected for a bunch charge around $190 \mathrm{pC}$ (corresponding to a peak current of roughly $3.3 \mathrm{~mA}$ ). Because of the performance limitations of the SRI FEA it was however not possible to experimentally observe the transition.

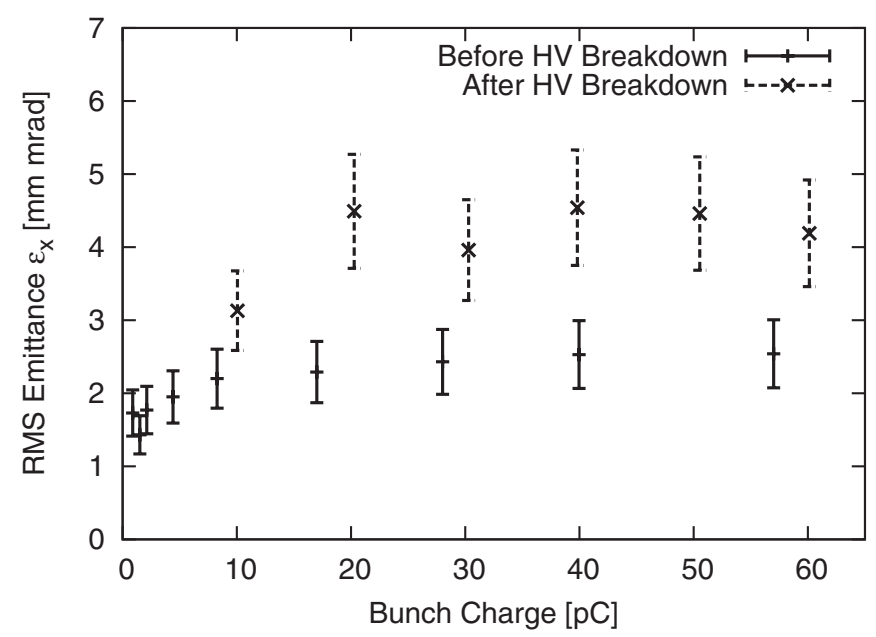

FIG. 7. Transverse rms emittance measured as a function of bunch charge. A significant emittance increase was observed after a HV breakdown incident; this is caused by inhomogeneous emission due to FEA surface damage.

\section{CONCLUSIONS}

The $100 \mathrm{keV}$ gun test stand has been successfully commissioned and is now available to characterize new and optimized FEA types as well as other potential cathode candidates. Although the commercially available SRI FEA is not suitable as a cathode for the low-emittance gun, it has been successfully used to commission the gun test stand and to show that the available diagnostic procedures are adequate to characterize FEA cathode beams.

The SRI FEAs have been characterized in terms of performance and transverse phase space distribution of the emitted bunches. While the projected transverse emittance is comparable to that of state-of-the-art FEL injectors, the peak current (and hence the brightness) is significantly lower $[15,16]$. The acquired emittance results allow an estimate of the emission cone opening angle at the FEA: it is on the order of $100 \mathrm{mrad}$. This clearly demonstrates the need for a double-gated FEA with a focusing layer to achieve the source emittance goal for the lowemittance gun.

PSI has started in-house development of a novel type of double-gated FEA with a metallic substrate and a high tip density [17]. The FEA is expected to have a lower bulk resistance and a reduced $\mathrm{RC}$ constant allowing shorter gate pulses and increased emission current. The source divergence will be minimized by the focusing layer thus reducing the source emittance. First samples are expected to arrive soon and will be benchmarked at the $100 \mathrm{keV}$ gun test stand. The increased current and reduced emittance will guarantee a space-charge dominated beam with which the emittance compensation scheme will be investigated. The next big step will be the construction of a $250 \mathrm{MeV}$ test facility with a FEA-based rf gun with high-gradient acceleration, solenoids for emittance compensation, linac and first magnetic compressor chicane to demonstrate feasibility of the acceleration concept for the compact $1 \AA$ X-FEL at PSI [18].

\section{ACKNOWLEDGMENTS}

We wish to acknowledge Romain Ganter for his advice regarding FEAs and Volker Schlott for his support with the diagnostics. We are grateful to Åke Andersson for his help during the commissioning of the test stand.

[1] D. Dowell, in Proceedings of the 2006 FEL Conference, Berlin, Germany, 2006, p. 22.

[2] R. Brinkmann, in Proceedings of the 2006 FEL Conference, Berlin, Germany, 2006, pp. 24-28.

[3] M. Böge, in Proceedings of EPAC 2002, Paris, France, 2002, pp. 39-43.

[4] An x-ray free electron laser at Paul Scherrer Institute, http://fel.web.psi.ch/public/publications/2005/ ESFRI\%20PSI-FEL.pdf. 
[5] R.J. Bakker et al., in Proceedings of the 2006 FEL Conference, Berlin, Germany, 2006, pp. 214-217.

[6] S. C. Leemann, Ph.D. thesis, EPF Lausanne, 2007, http:// library.epfl.ch/en/theses/?nr=3724.

[7] C. M. Tang, A.C. Ting, and T. Swyden, Nucl. Instrum. Methods Phys. Res., Sect. A 318, 353 (1992).

[8] CST GmbH, MAFIA, http://www.cst.com.

[9] Pulsar Physics, GPT, http://www.pulsar.nl/gpt.

[10] SRI International: MicroSystems Innovation Center, http://www.sri.com/psd/microsys/.

[11] R. H. Fowler and L. Nordheim, Proc. R. Soc. A 119, 173 (1928).

[12] K. L. Jensen, M. A. Kodis, R. A. Murphy, and E. G. Zaidman, J. Appl. Phys. 82, 845 (1997).

[13] A.E. Candel, Ph.D. thesis, ETH Zurich, 2005, http://www.itp.phys.ethz.ch/staff/candel/publications/
PhDThesis.pdf.

[14] M. Dehler, A. Candel, and E. Gjonaj, J. Vac. Sci. Technol. B 24, 892 (2006).

[15] M. Krasilnikov et al., in Proceedings of EPAC 2004, Lucerne, Switzerland, 2004, pp. 360-362.

[16] K. Togawa, T. Shintake, T. Inagaki, K. Onoe, T. Tanaka, H. Baba, and H. Matsumoto, Phys. Rev. ST Accel. Beams 10, 020703 (2007).

[17] E. Kirk, S. Tsujino, H. Sehr, T. Vogel, J. Gobrecht, R. Ganter, and A. Wrulich, in Eighth IEEE International Vacuum Electronics Conference, Kitakyushu, Japan, 2007.

[18] T. Schietinger et al., in Proceedings of the 22nd Particle Accelerator Conference, Albuquerque, New Mexico, 2007. 